\title{
Bilateral lung transplant for pulmonary hypertension with pulmonary artery aneurysm
}

\author{
Berhane Worku ${ }^{1}$, Charles Mack ${ }^{2}$, and Ivancarmine Gambardella ${ }^{2}$ \\ ${ }^{1}$ New York Presbyterian Weill Cornell Medicine \\ ${ }^{2}$ Weill Cornell Medical College
}

January 7, 2022

Bilateral lung transplant for pulmonary hypertension with pulmonary artery aneurysm

Berhane Worku MD ${ }^{1,2}$, Charles Mack MD ${ }^{1,3}$, Ivancarmine Gambardella $\mathrm{MD}^{1,2}$

1. New York Presbyterian Weill Cornell Medical Center, New York NY 10021

2. New York Presbyterian Brooklyn Methodist Hospital, Brooklyn NY 11215

3. New York Presbyterian Queens Hospital, Queens NY 11335

Corresponding Author

Berhane Worku MD Brooklyn Methodist Hospital

Department of Cardiothoracic Surgery

$5066^{\text {th }}$ Street

Brooklyn, NY 11215

718-780-7700

Bmw2002@med.cornell.edu

Pulmonary artery aneurysms (PAA) may be secondary to congenital cardiac defects such as a patent ductus arteriousus (PDA), atrial septal defect, or ventricular septal defect. They may also occur secondary to infection or connective tissue disease or they may be idiopathic in nature. Repair is undertaken to prevent the sequelae of rupture or dissection, although the specific size criteria at which repair is recommended remains controversial. Pulmonary hypertension $(\mathrm{PH})$ may also lead to PAA, in which case isolated repair is not recommended. Heart-lung transplant has classically been the treatment of choice for PH with PAA, especially when associated with congenital heart defects, right ventricular dysfunction, and pulmonic valve regurgitation.

In the setting of PH with PAA and correctable cardiac defects, bilateral lung transplant (BLT) has been described. Concurrent PAA repair is required, and several techniques exist to allow for this. In the current issue of the Journal of Cardiac Surgery, Doi et. al. offer a review of PH with PAA, with a focus on strategies to allow for BLT and PAA repair, hence avoiding the need for HLT. They describe a case of a patient with PH secondary to a PDA and a 9cm PAA who underwent BLT and PAA repair. The donor descending aorta and a bovine pericardial tube was used to reconstruct the recipient main and right PA, respectively. The patient suffered from persistently elevated PA pressures postoperatively due to a kink in the anastomosis between the neo-main PA (donor descending aorta) and the neo-right PA (bovine pericardial tube) requiring surgical revision, but the patient otherwise made an excellent recovery ${ }^{1}$. 
The benefit with BLT (rather than HLT) stems from limitations in donor supply which may result in unacceptably long wait times and reduced waitlist survival in patients awaiting HLT. As right ventricular function typically improves after BLT for PH, the donor heart from a HLT bloc may be better served to another patient with terminal cardiac failure. A variety of techniques have been described to allow for repair of massive PAAs at the time of BLT. Harvesting of the entire donor PA to allow replacement of the PAA has been described and is feasible when the donor heart is unsuitable for transplantation ${ }^{2,3}$. When the donor main PA is unavailable for harvesting, pulmonary arterioplasty and replacement with donor descending aorta have been described at the time of $\mathrm{BLT}^{4-7}$. After resection of the PAA, the proximal donor aorta is anastamosed to the proximal PA with the distal aorta oriented towards the right lung. The distal donor aorta is anastamosed to the donor right PA. The innominate and left carotid orifices can be used for anastomoses to the donor left $\mathrm{PA}^{5,6}$. Extension of a short donor left PA with an autologous pericardial tube has been described $^{5}$. Similarly, extension of a short donor right PA with a bovine pericardial tube is described in the current report ${ }^{1}$.

Pulmonary valve (PV) regurgitation may occur secondary to annular dilation from the PAA. PV replacement has been described, including sutureless valve implantation with valves intended for percutaneous deployment ${ }^{4}$. Durability remains a concern, and valve sparing repair techniques (commisuroplasty) have also been described ${ }^{3}$. When the donor heart is not being harvested, BLT with procurement of the donor right ventricular outflow graft has been described ${ }^{8}$. HLT always remains a reasonable option in the setting of extremely massive PAA associated with severe PV regurgitation and right ventricular dysfunction, assuming adequate donor availability and ability of the recipient to tolerate the longer wait time ${ }^{9}$.

Recovery of right ventricular function and tricuspid regurgitation after BLT for PH has been documented, supporting the shift from HLT to BLT for this entity. In the setting of left ventricular diastolic dysfunction, severe pulmonary edema and hypoxia can be seen after BLT for PH as the LV is suddenly loaded, and in such a scenario ECMO has been utilized to allow time for LV remodeling. Various centers may prefer HLT over BLT for these cardiac consequences of prolonged $\mathrm{PH}^{10}$. In the absence of these complicating factors, BLT should be considered for PH in otherwise appropriate candidates. BLT for PH with PAA is likely best managed with harvesting the donor main PA when the donor heart is not being considered for harvest. When the donor PA is not available, the decision to attempt the abovementioned strategies for PAA repair such as neo-PA creation with donor aorta and the associated prolongation of donor ischemic time must be weighed against exposing the patient to elevated waitlist mortality while waiting for an acceptable heart-lung bloc to become available. Transplant center expertise and regional differences in heart and lung donor utilization rates will likely a relevant factor to consider when selecting the optimal strategy for each patient.

\section{REFERENCES}

1. Doi A, Gajera J, Niewodowski D, Gangahanumaiah S, Whitford H, Snell G, Kaye D, Joseph T, McGriffin D. Surgical management of giant pulmonary artery aneurysms in patients with severe pulmonary arterial hypertension. J Card Surg; in press]

2. Schwarz S, Benazzo A, Prosch H, Jaksch P, Klepetko W, Hoetzenecker K. Lung transplantation for pulmonary hypertension with giant pulmonary artery aneurysm. J Thorac Cardiovasc Surg 2020;159:254350

3. Shayan H, Sareyyupoglu B, Shigemura N, Thacker J, Bermudez C, Toyoda Y. Lung transplant, double valve repair, and pulmonary artery aneurysm resection. Ann Thorac Surg 2012;93:e3-5

4. Pelenghi S, Primiceri C, Belliato M, Ghio S, Scelsi L, Totaro P. Is it time for a paradigm shift: Should double-lung transplant be considered the treatment of choice for idiopathic pulmonary arterial hypertension and giant pulmonary aneurysm? J Card Surg 2021;36:2996-2999

5. Noda M, Okada Y, Saiki Y, Sado T, Hoshikawa Y, Endo C, Sakurada A, Maeda S, Oishi H, Kondo $\mathrm{T}$. Reconstruction of pulmonary artery with donor aorta and autopericardium in lung transplantation. Ann Thorac Surg 2013;96:e17-9

6. Force SD, Lau CL, Moazami N, Trulock EP, Patterson GA. Bilateral lung transplantation and pulmonary artery reconstruction in a patient with chronic obstructive pulmonary disease and a giant 
pulmonary artery aneurysm. J Thorac Cardiovasc Surg 2003;126:864-6.

7. Oda H, Hamaji M, Motoyama H, Ikeda T, Minatoya K, Nakajima D, Chen-Yoshikawa TF, Date H. Use of a three-dimensional model in lung transplantation for a patient with giant pulmonary aneurysm. Ann Thorac Surg 2020;109:e183-5

8. Zanotti G, Hartwig MG, Davis RD. A simplified technique for pulmonary artery aneurysm repair in a lung transplant recipient with right ventricular outflow tract obstruction. J Thorac Cardiovasc Surg 2013;145: 295-6

9. Eadington T, Santhanakrishnan K, Venkateswaran. Heart-lung transplantation for idiopathic pulmonary arterial hypertension and giant pulmonary artery aneurysm - case report. J Cardiothorac Surg 2020;15:169

10. Budev MM, Yun JJ. Advanced circulatory support and lung transplantation in pulmonary hypertension. 